\title{
An Effective Method for Maximizing Social Welfare in Electricity Market via Optimal TCSC Installation
}

\author{
Thanh Long Duong \\ Faculty of Electrical Engineering Technology \\ Industrial University of Ho Chi Minh City \\ Ho Chi Minh City, Vietnam \\ duongthanhlong@iuh.edu.vn
}

Ngoc Anh Nguyen

Faculty of Electrical Engineering Technology Industrial University of Ho Chi Minh City Ho Chi Minh City, Vietnam

nguyenngocanh@iuh.edu.vn

\author{
Thuan Thanh Nguyen \\ Faculty of Electrical Engineering Technology \\ Industrial University of Ho Chi Minh City \\ Ho Chi Minh City, Vietnam \\ nguyenthanhthuan@iuh.edu.vn \\ Le Anh Tu Nguyen \\ Faculty of Electrical Engineering Technology \\ Industrial University of Ho Chi Minh City \\ Ho Chi Minh City, Vietnam \\ lenguyenanhtu@iuh.edu.vn
}

\begin{abstract}
The evolving electricity market has increased power demand and has brought many social benefits. Meanwhile, the transmission systems are not developed to the same extent because building new lines is difficult for environmental and political reasons. Hence, the systems are driven close to their limits resulting in congestions and critical situations endangering the system's security. Due to this, the study of enhancing the transfer capability of existing power networks to satisfy the increased power demand, maximum social welfare, and ensure its secure operation has become one of the challenges the Independent System Operator faces in the electricity market. In order to solve this problem without building more transmission lines, the installation of FACTS devices can be a better alternative. Among FACTS devices, thyristor-controlled series compensator (TCSC) is one that can redistribute power flow in the network to improve the transfer capability of the existing system effectively. However, it is very difficult to implement. This paper presents the implementation of the Cuckoo Optimization Algorithm (COA) to solve the OPF problem which is formulated as a nonlinear optimization problem with equality and inequality constraints in a power system for social welfare maximization via the optimal installation of TCSC devices. As Cuckoo Search Algorithm (CSA), the COA also starts with an initial populationbased metaheuristic optimization inspired by the nature of brood parasitism of some cuckoo species. However, unlike CSA, COA uses the cuckoo's style for egg-laying to optimize the local search instead of using Lévy flights. This model is tested in IEEE 14 and IEEE 30 bus systems. Simulations results are compared with GA and GWO and show that the COA is one useful method for TCSC installation to maximize social welfare.
\end{abstract}

Keywords-cuckoo optimization algorithm; FACTS; TCSC; social benefits; electricity market

\section{INTRODUCTION}

Due to the rapid technological progress, the consumption of electric energy increases continuously. For the last three decades, many electrical power utilities have been forced to change their way of operation from monopolistic structure to competitive market structure. In Vietnam, pilot steps of the roadmap for the application of electricity market approved by the Prime Minister, are being applied and the country moves towards a competitive electricity market. The basic idea of deregulation is to make supply and demand competitive so that all participants, i.e. generator companies, distribution companies, and the customers maximize their individual welfare. The supply or cost bid provided by a generator company is the minimum asking price that it would accept for supplying a particular amount of power. Similarly, the demand or benefit bid of a consumer is the maximum price that it would pay for consuming a particular amount of power. The Independent System Operator (ISO) is responsible for the buying and selling of power between generator and distribution companies to the customers based on supply and demand bids, which are prepared in such a way as to maximize social welfare. In order to solve this problem, transmission improvement methods are applied to achieve minimum generation costs, subject to system security constraints. In contrast, social welfare maximizing is the objective of most studies in deregulated power systems. Several studies have also been performed on congestion management to maximize social and individual welfare [1-2], as well as social welfare maximization considering reactive power and congestion management in the deregulated environment [3]. Distributed generator locations for social welfare maximization are presented in [4]. These studies focused on a method for increasing social welfare under congestion probability in transmission networks.

Recently, Flexible AC Transmission Systems (FACTS) [5] have also been utilized to solve the congestion problem and maximize social welfare. Many studies have been proposed for the improvement of existing power networks via optimal location of FACTS [6-19]. Interior point method was used for system expansion with UPFC to maximize social welfare and 
to manage congestion [20]. In [21], Mixed Integer Non-linear programming was used for optimal location of FACTS to maximize social welfare based on multiple time periods. But it ignored the impact of FACTS on the reactive power flow. In [22] FACTS device thyristor-controlled series compensator (TCSC) is optimally located for congestion management maximizing social welfare using genetic algorithm (GA) and GWO algorithm. An efficient GA was proposed for optimal size and location of TCSC in a deregulated market for congestion management with aim of maximizing the social welfare cost [23]. In [24], a new fuzzy-based genetic algorithm (Fuzzy-GA) for alleviating congestion and maximizing social benefit in a double-sided auction market by locating and sizing of one TCSC unit. In [25], a GA for finding the optimal location and size of this device was proposed for congestion management with the aim of increasing social welfare. A novel efficient differential evolution and evolutionary programming technique for social welfare maximization by optimal location of various FACTS devices in pool electricity market based on contingency analysis was introduced in [26].

Optimal location of FACTS devices is a complex combinatorial analysis, which is best studied using metaheuristic algorithms. Many researchers have tested different optimization algorithms. A new meta-heuristic algorithm called Cuckoo Optimization Algorithm (COA) was developed in [27]. COA is a nature-inspired meta heuristic algorithm which is inspired by the life of cuckoo bird family. The special lifestyle of these birds and their characteristics in egg-laying and breeding has been the basic motivation for development of this new evolutionary optimization algorithm. It is a novel evolutionary algorithm, suitable for the continuous nonlinear optimization problem. Similar to other evolutionary methods, the initial population of COA is randomly generated within the control parameter limits. The effort to survive among cuckoos constitutes the basis COA. During the survival competition some of the cuckoos or their eggs may perish. The survived cuckoo societies migrate to a better environment and start reproducing and laying eggs. Cuckoos survival effort hopefully converges to a state that there is only one cuckoo society, with all birds having the best profit values. Application of the COA algorithm to some benchmark functions and a real problem has proven its capability in solving complex, nonlinear and non-convex optimization problems [27]. The key features of the $\mathrm{COA}$ are its faster convergence rate and the reduction in computational complexity. Hence, it may become an effective tool in solving power system optimization problems. However, the COA is only introduced in the OPF problem [28], and it still hasn't been used to solve TCSC optimization problems. In this paper, COA has been implemented to solve the OPF problem which is formulated as a nonlinear optimization problem with equality and inequality constraints in a power system for maximization of the social welfare via optimal installation of TCSC devices. The proposed approach has been tested on the IEEE 14-bus and IEEE 30-bus systems. Simulation results are compared with the re results of GA and GWO and show that the COA is also one of useful algorithm in solving the power system optimization problem.

\section{PROBLEM FORMULATION}

\section{A. Modeling of TCSC}

The model of the network with TCSC is shown in Figure 1. TCSC is integrated in the OPF problem by modifying the line data. This device may have either inductive compensation or capacitive compensation by limiting $70 \%$ to $50 \%$ of the reactance of the uncompensated line where TCSC is located. Let $X_{i j}$ be the reactance of the transmission line and $X_{T C S C}$ the reactance of TCSC and $X_{n e w}$ the new reactance of the line after placing TCSC between buses $i$ and $j$ :

$$
\begin{gathered}
X_{N e w}=X_{i j}+X_{T C S C} \\
X_{T C S C}=k_{T C S C} X_{i j} \\
P_{i j}=V_{i}^{2} G_{i j}-V_{i} V_{j}\left(G_{i j} \cos \delta_{i j}+B_{i j} \sin \delta_{i j}\right) \\
Q_{i j}=-V_{i}^{2} B_{i j}-V_{i} V_{j}\left(G_{i j} \sin \delta_{i j}-B_{i j} \cos \delta_{i j}\right) \\
P_{j i}=V_{j}^{2} G_{i j}-V_{i} V_{j}\left(G_{i j} \cos \delta_{i j}-B_{i j} \sin \delta_{i j}\right) \\
Q_{j i}=-V_{j}^{2} B_{i j}+V_{i} V_{j}\left(G_{i j} \sin \delta_{i j}+B_{i j} \cos \delta_{i j}\right)
\end{gathered}
$$

where $\delta_{i j}$ is the voltage angle difference between bus $i$ and bus,; the conductance and susceptance of transmission line can be calculated respectively as:

$$
\begin{aligned}
G_{i j} & =\frac{R_{i j}}{R_{i j}^{2}+X_{\text {new }}^{2}} \\
B_{i j} & =\frac{X_{\text {new }}}{R_{i j}^{2}+X_{i j}^{2}}
\end{aligned}
$$

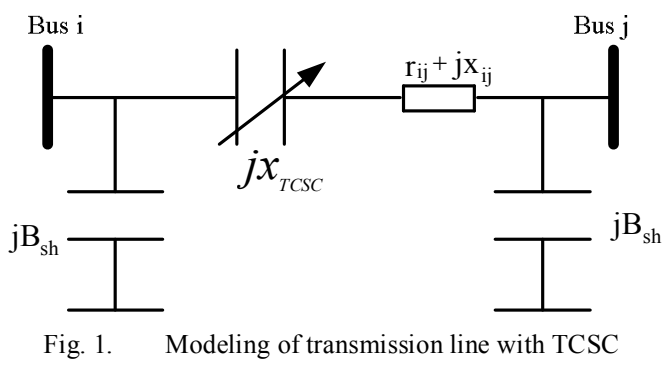

\section{B. Objective Function}

Market prices for a given amount of power can be determined by solving the optimization problem with the objective of maximizing social welfare to satisfy limited constraints. Generally, bulk loads as well as retailers in deregulated electricity market are required to bid their maximum demand and price function. Similarly, all generators are also required to bid their generation cost function along with their maximum generation. In electricity market, generation and distribution companies are allowed to offer and bid their prices to the ISO [29]. Social welfare optimization provides the demand and the generation of all the buses to be known. Let the vector of pool real power demand $\left(\underline{P}_{d p}\right)$ and 
vector of pool real power generation $\left(P_{g p}\right)$ be given in $(9)$ and (10):

$$
\begin{aligned}
P d^{p} & =\left\{P d_{j}^{P} ; j=1,2,3 \ldots . . m\right\} \\
P g^{p} & =\left\{P g_{i}^{P} ; i=1,2,3 \ldots . . n\right\}
\end{aligned}
$$

where, $m$ is the number of dispatch able loads, and $n$ is the number of generators. Let the generation cost curve of the generator placed at bus $i$ bidding to the pool be denoted by $C_{i}\left(P g_{i}^{P}\right)$. The benefit function for a load is $B_{j}\left(P d_{j}^{P}\right)$. It represents the load price which is willing to pay for the purchase of an amount of electric power $P d{ }_{j}^{p}$. In electricity market, the social benefit for an elastic load is given by (11). The elastic load is sensitive to the energy price.

$$
\operatorname{Max}\left(\sum_{i \in N_{g}} W_{i}\left(P g_{i}\right)+\sum_{i \in N_{d}} W_{j}\left(P d_{j}\right)\right)
$$

where $W_{j}\left(P_{d j}\right)$ is the consumers welfare function. Optimal social welfare occurs when the total of all generator and consumer units' welfare in the system are maximized.

$$
W_{j}\left(P d_{j}\right)=B_{j}\left(P d_{j}\right)-\lambda P d_{j}
$$

The $B_{j}$ in (12) indicates the $i$-th load utility function and $\lambda$ is the amount of cost that should be paid for each MW of energy per unit. $W_{i}\left(P_{g i}\right)$ is the welfare function of the generators:

$$
W_{i}\left(P g_{i}\right)=\lambda P g_{i}-C_{i}\left(P g_{i}\right)
$$

$C_{i}$ is representing the cost function of the $i$-th generator. The $\lambda$ is the cost each generator should be paid per MW of energy. From (11)-(13) the objective function can be written as:

$$
F_{o b j}=\operatorname{Max}\left(\sum_{i \in N_{d}} B_{j}\left(P d_{j}\right)-\sum_{i \in N_{g}} C_{i}\left(P g_{i}\right)\right)
$$

where, $C_{i}\left(P g_{i}^{P}\right)=a_{g i} P_{g i}^{2}+b_{g i} P_{g i}+c_{g i}$ is the cost function of the generator and $B_{j}\left(P d_{j}^{P}\right)=a_{d j} P_{d j}^{2}+b_{d j} P_{d j}+c_{d j}$ is the profit function of the customer.

The social welfare is mathematically maximized subject to the following transmission network constraints:

- Power balance equation:

$$
\begin{array}{lc}
P_{i}(V, \delta)+P_{d i}-P_{g i}=0 & i=1, \ldots, N_{b} \\
Q_{i}(V, \delta)+Q_{d i}-Q_{g i}=0 & i=1, \ldots, N_{b}
\end{array}
$$

- Power generation limit :

$$
\begin{aligned}
& P_{g i}^{\min } \leq P_{g i} \leq P_{g i}^{\max } \quad i=1, \ldots ., N_{g} \\
& Q_{g i}^{\min } \leq Q_{g i} \leq Q_{g i}^{\max } \quad i=1, \ldots ., N_{g}
\end{aligned}
$$

- Bus voltage limits:

$$
V_{i}^{\min } \leq V_{i} \leq V_{i}^{\max } \quad i=1, \ldots ., N_{b}
$$

- Apparent line flow limit:

$$
S_{l} \leq S_{l, \max } \quad l=1, \ldots ., N_{l}
$$

\section{INTRODUCTION OF COA}

COA is inspired by the special life style of cuckoo birds. No cuckoo bird nests its eggs. Mature cuckoos have to find a host bird nest to safely place their eggs. After that, the feed responsibility belongs to the host bird. Only a number of the cuckoo's eggs have the chance to grow and become mature cuckoos. All mature cuckoos will move forward to the best Habitat. After some iterations, the cuckoo populations will converge in a Habitat with the best profit values [27]. Like other evolutionary algorithms, the proposed algorithm starts with an initial population of cuckoos. These initial cuckoos lay some eggs in some host bird's nests. Some of these eggs which are more similar to the host bird's eggs have the opportunity to grow and become mature cuckoos. Other eggs are detected by the host birds and are destroyed. The grown eggs reveal the suitability of the nests in that area. The more eggs survive in an area, the more profit is gained in that area. So, the Habitat in which more eggs survive will be the term that COA is going to optimize.

\section{A. Generating the Initial Cuckoo Habitat}

In order to solve an optimization problem, it is necessary the values of problem variables to be formed as an array. In COA, it is called Habitat. In the $N_{\text {var }}$ dimensional optimization problem, a Habitat is an array of $1 \times N v a r$, representing the current living Habitat of cuckoo. This array is defined as:

$$
\text { Habitat }=[x 1, x 2, \ldots, x \text { Nvar }]^{\mathrm{T}}
$$

Each of the variable values $(x 1, x 2, \ldots, x N v a r)$ is a floating point number and a Habitat may represent a vector of control variables of the OPF problem. The profit of a habitat is obtained by the evaluation of the profit function $f_{P}$ at a Habitat.

$$
\text { Profit }=f_{p}(\text { Habitat })=\mathrm{f}_{\mathrm{p}}(x 1, x 2, \ldots, x N v a r)
$$

$\mathrm{COA}$ is an algorithm that maximizes the profit function. In order to use COA in cost minimization problems such as minimizing the fitness function in the OPF problem, (22) can be write as:

$$
\text { Profit }=-\operatorname{Cost}(\text { Habitat })=-f_{p}(x 1, x 2, \ldots, x \text { Nvar })(23)
$$

\section{B. Cuckoo's Style for Egg Laying}

In the first iteration, a candidate Habitat matrix of size $N_{P o p} \times N_{V a r}$ is generated. Then some random eggs are dedicated to each cuckoo and its ELR (Eggs Laying Radius) is calculated. The ELR is defined as:

$$
\text { ELR }=\alpha * \frac{\text { Number of current Cuckoo's eggs }}{\text { Total number of eggs }} *\left(v a r_{h i}-\text { var }_{\text {low }}\right)
$$

where $\alpha$ is an integer to handle the maximum value for ELR and $v a r_{h i}$ and $v a r_{l o w}$ is the up and down limits of optimal variables. The aim of ELR is to determine and limit the searching space in each iteration step. 


\section{Eliminating Cuckoos in Worst Habitats}

Due to the equilibrium in bird's population, only a maximum number of cuckoos may live in the environment, termed as MaxNumofCuckoos. A priority list may be created by evaluation and sorting of the Profit value of the Habitat of each newly grown cuckoo. So, there are MaxNumofCuckoos cuckoos from the first of priority list alive, and the other cuckoos will die. This work will decrease computational time because there is a limited number of best solutions to be used in the next iteration.

\section{Immigration of Cuckoos}

The Habitat in which cuckoos have the best conditions to live and grow or the best profit value will be considered as the goal point of the other cuckoos. They will immigrate as closer as they can towards the goal point. After a period of time, cuckoos grow to mature cuckoos and restart the process. This means that cuckoo population will find the best place to live after many iterations. This work will help the OPF problem to find the best solutions.

\section{APPLICATION COA FOR SOLVING THE PROBLEM OF SOCIAL WELFARE MAXIMIZATION}

The flow chart of COA for the social welfare maximization problem is presented in Figure 2.

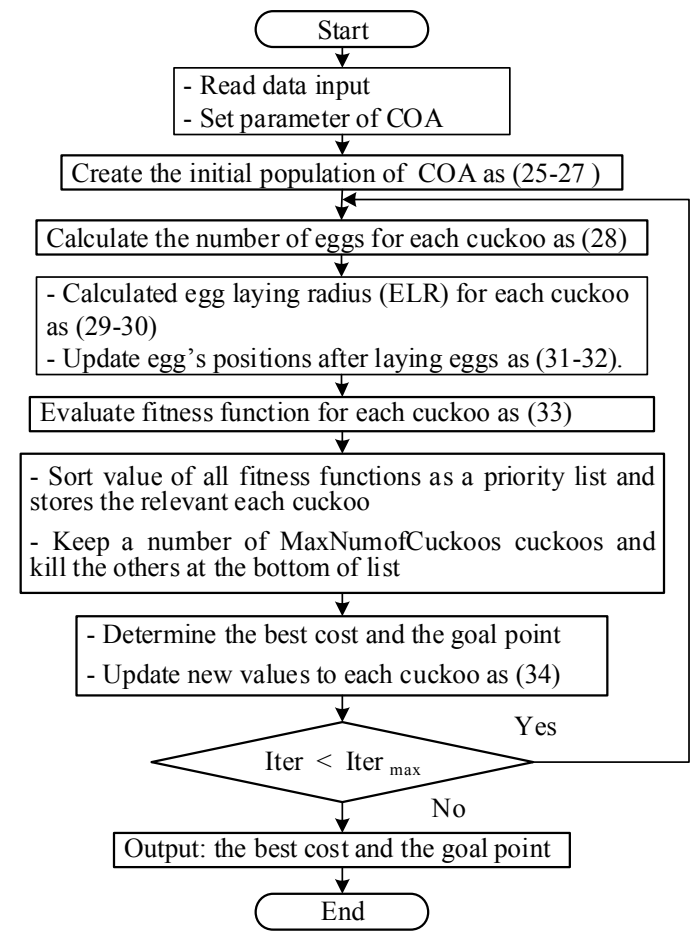

Fig. 2. The flowchart of COA for the social welfare maximization problem

The steps of the maximizing social welfare problem using the proposed COA are presented below.

Step 1: Prepare a system data base. System topology, line and load specifications, generation limits, line flow limits and cost coefficient parameters.
Step 2: Set parameter for COA and social welfare problem: NumCuckoos: the number of cuckoos in the first population, MinEggs: the minimum number of eggs of each cuckoo, MaxEggs: the maximum number of eggs of each cuckoo, MaxIter: the maximum number of iterations, Motion Coceff: the variable to control the towards to goal point process, MaxCuckoos: the maximum number of Cuckoos that can live at the same time, RadiusCoceff: the control parameter of egg laying radius, $N_{p a r}$ is the number of optimal variables. It is set equal to the number of variables included in vector $X_{i d} . K_{p}, K_{q}$, $K_{v}, K_{s}$ : the penalty factors.

Step 3: Create the initial population:

$$
X_{i d}=\left[P_{g 2} \ldots P_{g N g}, P_{d 2} \ldots . P_{d N d}, V_{g 1} \ldots V_{g N g}, N_{T C S C}, K_{T C S C}\right]
$$

Each Habitat of cuckoos $\left(X_{i d}\right)$ is created as follows:

$$
X_{i d}=X_{i d}^{\min }+\operatorname{rand} 1 *\left(X_{i d}^{\max }-X_{i d}^{\min }\right)
$$

A random goal point is created as follow:

$$
\text { GoalPoint }=X_{i d}^{\min }+\operatorname{rand} 1 *\left(X_{i d}^{\max }-X_{i d}^{\min }\right)
$$

The Habitat is a random matrix sized $\left[1 \times N_{p a r}\right]$, and is representing the living environment of each cuckoo.

Step 4: Calculate the number of eggs for each cuckoo as:

$$
\begin{aligned}
& \text { NumberCuckoo's eggs }= \\
& (\text { MaxEggs }- \text { MinEggs }) \text { xrand } 2+\text { MinEggs }
\end{aligned}
$$

Step 5: Calculate the ELR for each cuckoo. Update egg's positions after laying eggs.

$$
\begin{gathered}
E L R_{i}^{\max }=\frac{\text { numbercuckoo's eggs } *\left(X_{i d}^{\max }-X_{i d}^{\min }\right) * \text { RadiusCoeff }}{\text { Total Number of egg }} \\
E L R_{i}=E L R_{i}^{\max } * \text { rand } 3
\end{gathered}
$$

where rand 3 is a random matrix with size $1 x$ Number of current eggs, so $E L R_{i}$ is a matrix with number of eggs rows and $N_{p a r}$ columns.

$$
\begin{gathered}
A d d_{i}=\left((-1)^{\text {rand } 4} * E L R_{i} * \cos \operatorname{in}(\text { angles })+E L R_{i} * \sin (\text { angles })\right) \\
X_{i d}^{(1)}=X_{i d}+A d d_{i}
\end{gathered}
$$

where rand 4 is a random value, it can be set to 1 or 2 , and angles is a random line space represent for the flying angles of cuckoo. Each row of matrix $X_{i d}$ is a candidate for the vector habitat $X_{i d}$. Then, the limit for each $X_{i d}$ is checked and the egg laying process is done.

Step 6: Solve power flow for each candidate $X_{i d}$. The fitness function is calculated by:

$$
\begin{aligned}
& F_{f}=F_{o b j}-k_{p} \sum_{i=1}^{N B}\left(P_{g i}-P_{g i}^{\lim }\right)^{2}-k_{q} \sum_{i=1}^{N B}\left(Q_{g i}-Q_{g i}^{\lim }\right)^{2} \\
& -k_{V} \sum_{i=1}^{N B}\left(V_{i}-V_{i}^{\lim }\right)^{2}-k_{S} \sum_{i=1}^{N L}\left(s_{l i}-S_{l i}^{\max }\right)^{2}
\end{aligned}
$$


Step 7: Evaluate the fitness function for each $X_{i d}$ in Step 6. Sort the value of all fitness functions as a priority list and store the relevant $X_{i d}$.

Step 8: Based on the priority list, keep a number of MaxNumofCuckoos $X_{i d}$ and kill the others at the bottom of the list.

Step 9: The first $X_{i d}$ of the list is the best $X_{i d}$ solution in this iteration. It is the best cost and will be the new goal point for the others. Their new Habitat is determined as:

$$
X_{i d}^{(2)}=\left(\text { Goalpo int }-X_{i d}^{(1)}\right) * \text { rand } 5 * \text { MotionCoeff }+X_{i d}^{(1)}
$$

Step 10: Update new values to $X_{i d}$ and save the best cost, which is the goal point.

Step 11: Check the condition to stop the program. If $I_{\text {ter }}<I_{\text {terMax }}, I_{\text {ter }}=I_{\text {ter }}+1$ and return to Step 4. Otherwise, stop.

\section{NUMERICAL RESULTS}

\section{A. IEEE 14-Bus Test System}

The IEEE 14-bus system was used to investigate the effect of TCSC on social welfare. The IEEE 14 bus system consists of 5 generators and 20 lines, as shown in Figure 3. The marginal benefit function for generator and load are given in [12]. Optimal power flow programs are executed by modifying the MATPOWER [30] program implementation of COA in scheduling GENCOS and DISCOS (Table I).

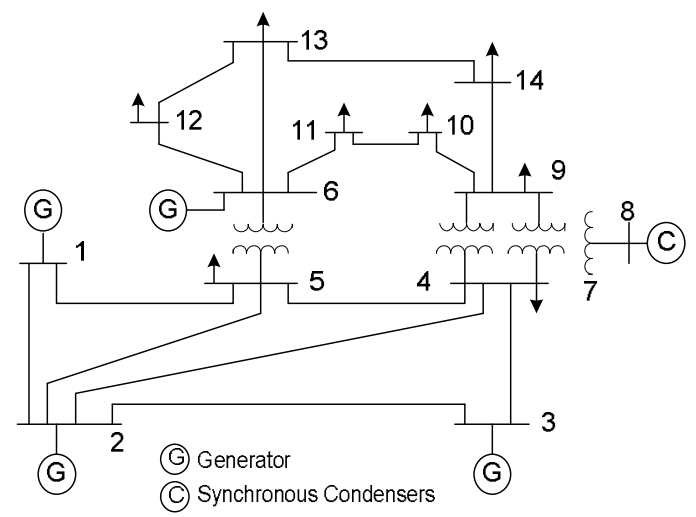

Fig. 3. The IEEE 14-bus system

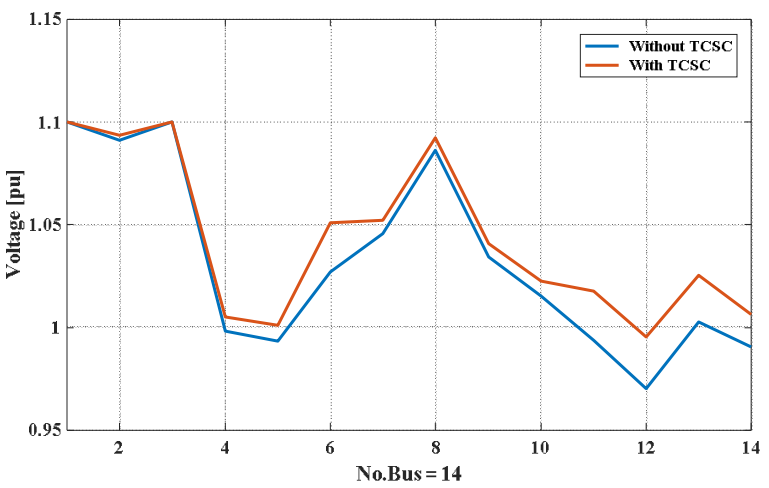

Fig. 4. The bus voltage profile of IEEE 14-bus system with and without TCSC

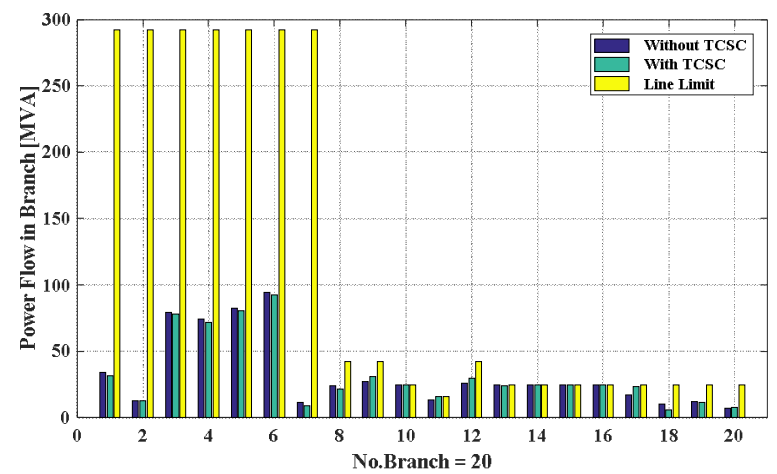

Fig. 5. Power flow branch of IEEE 14-bus system with and without TCSC

The results of social benefit, scheduling of GENCOS and DISCOS in the case of with and without TCSC for the IEEE 14 bus system are presented in Tables I-IV. From Table I it can be seen that, the total real power of the generator and the total power consumption in the case of using the COA algorithm are higher than when using the GA and GWO algorithm [22]. Moreover, the distribution of power consumption at the load buses of the COA algorithm compared to GA, GWO is also different, reflecting in better social benefit value for COA algorithm as shown in Table III.

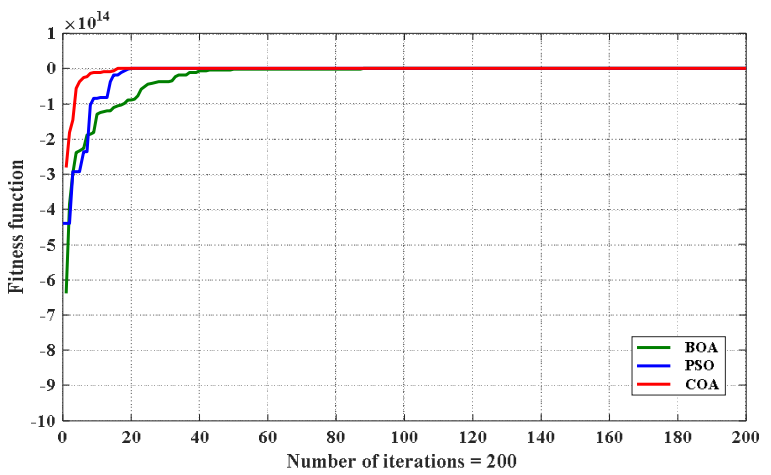

Fig. 6. Convergence characteristics of COA compared PSO and BOA for the IEEE 14 bus system without TCSC

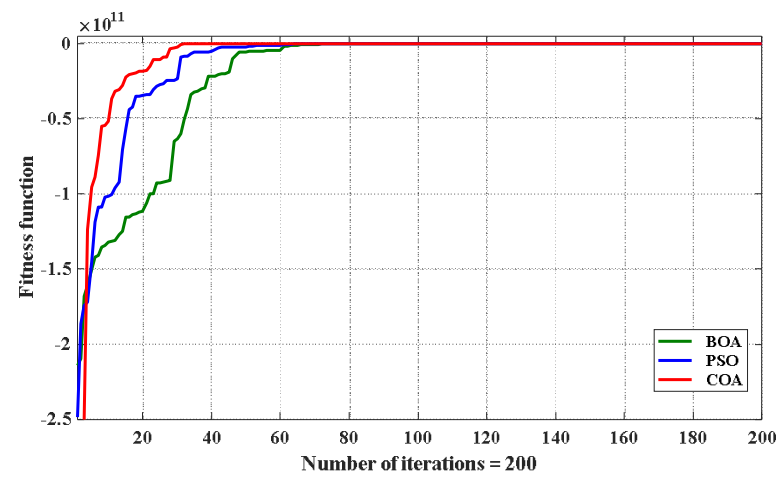

Fig. 7. Convergence characteristics of COA compared PSO and BOA for the IEEE 14 bus system with TCSC 
TABLE I. COMPARISON OF GA AND GWO SCHEDULING OF GENCOS AND DISCOS WITHOUT TCSC FOR THE IEEE 14 BUS SYSTEM

\begin{tabular}{|c|c|c|c|c|c|c|c|c|c|c|}
\hline & \multirow{2}{*}{$\begin{array}{c}\text { Bus } \\
\text { no }\end{array}$} & \multicolumn{3}{|c|}{ GA [22] } & \multicolumn{3}{|c|}{ GWO [22] } & \multicolumn{3}{|c|}{ COA } \\
\hline & & $\mathrm{V}(\mathrm{pu})$ & P (MW) & Q (Mvar) & $\mathrm{V}(\mathbf{p u})$ & P (MW) & Q (Mvar) & $\mathbf{V}(\mathbf{p u})$ & P (MW) & Q (Mvar) \\
\hline \multirow[t]{5}{*}{ Gen } & 1 & 1.1 & 94.97 & 45.43 & 1.1 & 97.55 & 43.51 & 1.1 & 97.675 & 39.895 \\
\hline & 2 & 1.089 & 100.0 & 56.58 & 1.089 & 100.0 & 54.57 & 1.091 & 100.00 & 59.818 \\
\hline & 3 & 1.095 & 100.0 & 29.86 & 1.095 & 100.04 & 29.18 & 1.100 & 100.00 & 34.050 \\
\hline & 6 & 1.035 & 52.08 & 7.22 & 1.035 & 50.31 & 15.29 & 1.027 & 52.176 & 10.825 \\
\hline & 8 & 1.09 & 0 & 25.28 & 1.09 & 0 & 25.21 & 1.086 & 0 & 24.995 \\
\hline Total & & & 347.0 & 164.67 & & 347.9 & 167.76 & & 349.8 & 169.58 \\
\hline \multirow[t]{8}{*}{ Dis } & 4 & 0.997 & 110.9 & 53.75 & 0.998 & 110.9 & 53.73 & 0.998 & 110.11 & 53.330 \\
\hline & 5 & 0.991 & 131.3 & 63.61 & 0.994 & 117.6 & 56.99 & 0.993 & 118.79 & 57.536 \\
\hline & 9 & 1.039 & 5.01 & 2.43 & 1.04 & 5.02 & 2.43 & 1.045 & 0 & 0 \\
\hline & 10 & 1.019 & 20.82 & 10.08 & 1.022 & 11.15 & 5.4 & 1.034 & 5.0015 & 2.4223 \\
\hline & 11 & 1.01 & 18.19 & 8.81 & 1.0 & 29.92 & 14.49 & 1.015 & 13.897 & 6.7306 \\
\hline & 12 & 0.998 & 18.51 & 8.97 & 0.978 & 31.35 & 15.18 & 0.993 & 28.420 & 13.764 \\
\hline & 13 & 1.01 & 14.42 & 6.98 & 1.01 & 5.63 & 2.73 & 0.970 & 31.485 & 15.249 \\
\hline & 14 & 1.009 & 12.07 & 5.85 & 0.998 & 19.51 & 9.45 & 1.002 & 5.0000 & 2.4216 \\
\hline Total & & & 331.3 & 160.49 & & 331.16 & 160.39 & & 332.83 & 161.20 \\
\hline
\end{tabular}

TABLE II. COMPARISON OF GA AND GWO SCHEDULING OF GENCOS AND DISCOS WITH TCSC FOR THE IEEE 14 BUS SYSTEM

\begin{tabular}{|c|c|c|c|c|c|c|c|c|c|c|}
\hline & \multirow{2}{*}{$\begin{array}{c}\text { Bus } \\
\text { no }\end{array}$} & \multicolumn{3}{|c|}{ GA [22] } & \multicolumn{3}{|c|}{ GWO [22] } & \multicolumn{3}{|c|}{ COA } \\
\hline & & V (pu) & $\mathbf{P}(\mathrm{MW})$ & Q (Mvar) & $V(p u)$ & $\mathbf{P}(\mathrm{MW})$ & Q (Mvar) & $V(p u)$ & P (MW) & Q (Mvar) \\
\hline \multirow{5}{*}{ Gen } & 1 & 1.1 & 98.37 & 53.65 & 1.1 & 93.83 & 44.26 & 1.1 & 94.903 & 32.391 \\
\hline & 2 & 1.089 & 100.1 & 53.66 & 1.089 & 101.1 & 52.95 & 1.093 & 100.03 & 59.480 \\
\hline & 3 & 1.095 & 100.0 & 30.1 & 1.095 & 101.1 & 28.42 & 1.099 & 100.00 & 28.517 \\
\hline & 6 & 1.035 & 64.25 & 7.92 & 1.035 & 49.8 & 15.42 & 1.051 & 49.492 & 19.235 \\
\hline & 8 & 1.09 & 0 & 25.49 & 1.09 & 0 & 26.0 & 1.092 & 0 & 24.987 \\
\hline Total & & & 362.8 & 170.82 & & 344.8 & 344.87 & & 344.43 & 164.61 \\
\hline \multirow[t]{8}{*}{ Dis } & 4 & 0.997 & 125.4 & 60.74 & 0.999 & 102.8 & 49.79 & 1.000 & 116.8 & 56.60 \\
\hline & 5 & 0.995 & 129.1 & 62.54 & 0.994 & 118.7 & 57.71 & 0.997 & 117.6 & 56.97 \\
\hline & 9 & 1.039 & 5.0 & 2.42 & 1.036 & 5.0 & 2.42 & 1.038 & 5.001 & 2.422 \\
\hline & 10 & 1.019 & 20.12 & 9.74 & 1.019 & 14.46 & 7.0 & 1.019 & 22.14 & 10.72 \\
\hline & 11 & 1.007 & 20.48 & 9.92 & 1.001 & 26.95 & 13.05 & 1.014 & 20.13 & 9.749 \\
\hline & 12 & 0.995 & 27.99 & 13.55 & 0.981 & 27.71 & 13.42 & 1.014 & 22.03 & 10.67 \\
\hline & 13 & 1.012 & 10.5 & 5.09 & 1.002 & 8.57 & 4.15 & 1.019 & 11.45 & 5.546 \\
\hline & 14 & 1.016 & 7.69 & 3.72 & 0.985 & 24.14 & 11.69 & 0.996 & 22.85 & 11.06 \\
\hline Total & & & 346.3 & 167.74 & & 328.37 & 159.04 & & 328.1 & 158.88 \\
\hline
\end{tabular}

TABLE III. COMPARISON OF GA, GWO AND COA FOR SOCIAL WELFARE MAXIMIZATION FOR THE IEEE 14 BUS SYSTEM

\begin{tabular}{|c|c|c|c|c|c|c|}
\hline & \multicolumn{3}{|c|}{ Without TCSC } & \multicolumn{3}{c|}{ With TCSC } \\
\cline { 2 - 7 } & GA [22] & GWO [22] & COA & GA [22] & GWO [22] & COA \\
\hline Gencos & 1408.93 & 1415.50 & 1424.87 & 1494.77 & 1401.62 & 1396.48 \\
\hline Discos & 2910.75 & 2966.62 & 2982.17 & 3014.85 & 2969.66 & 3022.30 \\
\hline Social Welfare & 1501.81 & 1551.12 & 1557.30 & 1520.08 & 1568.03 & 1581.21 \\
\hline \multicolumn{3}{|l}{ Location of TCSC } & Line 1-5 & Line 6-13 & Line 7-9 \\
\hline
\end{tabular}

Table III shows that the social benefit of GA and GWO algorithms is $1501.81(\$ / \mathrm{h})$ and $1551.12(\$ / \mathrm{h})$ while for COA is $1557.30(\$ / \mathrm{h})$. Compared with GA and GWO, the social benefit of COA is $3.56 \%$ higher than the one for GA and $0.39 \%$ higher than the one for GWO. In addition, as can be seen from Figures $4-5$, the power on the branches and the voltage at the buses when using the COA algorithm are within the allowable limits. The scheduling of GENCOS and DISCOS in the case with TCSC of COA are presented in Table II. The TCSC has redistributed the power flow on the lines, increasing social benefits. The optimal position of TCSC is at line 7-9 with $X_{T C S C}=-0.693 \mathrm{pu}$ when using the COA algorithm. From Figures $3-4$ it can be seen that the power flow on the branches in the system is redistributed with more balance, the voltage at the buses is also within limits after installing TCSC. Social benefits of GA, GWO and COA algorithms with TCSC are presented in Table III. It can be seen that the social benefit in the case when using TCSC of COA algorithm is 3.86\% higher than with GA algorithm with TCSC and $0.83 \%$ higher than with GWO algorithm with TCSC. The results of generator power and load power cases with and without TCSC using COA algorithm are presented in Table IV. From this Table, it can be seen that the total real power of generator and total power consumption in the case of TCSC is less than in the case without TCSC, but the social benefits in the case of TCSC are higher than without TCSC as shown in Table III. This shows that TCSC has 
improved social benefits. The TCSC has redistributed the power flow and improved the transmission capability. Hence, load buses that have high benefit factor tend to increase the power consumption as seen in Table IV (bus 10, bus 14), while load buses that have low benefit tend to reduce power consumption as seen in Table IV (bus 11, bus 4), so customer benefits are increased and results of social benefits in the case with TCSC is $1.51 \%$ higher than when there is no TCSC. In addition, the convergence of the COA algorithm is compared to Particle Swarm Optimization (PSO) and Butterfly Optimization Algorithm (BOA). From Figures 6-7 can be seen that COA has the ability to converge quickly when compared to PSO and BOA. This shows that the COA algorithm is able to find the suitable location and size of TCSC to improve social benefits in the electricity market. It also shows the ability of the proposed COA algorithm to converge.

TABLE IV.

THE RESULTS OF SCHEDULING OF GENCOS AND DISCOS WITH AND WITHOUT TCSC FOR THE IEEE 14 BUS SYSTEM

\begin{tabular}{|c|c|c|c|c|c|c|c|}
\hline \multirow{2}{*}{ COA } & \multirow[b]{2}{*}{ Bus no } & \multicolumn{3}{|c|}{ Without TCSC } & \multicolumn{3}{|c|}{ With TCSC } \\
\hline & & Voltage (pu) & Real power (MW) & Reactive power (Mvar) & Voltage (pu) & Real power (MW) & Reactive power (Mvar) \\
\hline \multirow[t]{5}{*}{ Gencos } & 1 & 1.1 & 97.6759 & 39.8956 & 1.1 & 94.9034 & 32.3914 \\
\hline & 2 & 1.0911 & 100.0001 & 59.8188 & 1.0935 & 100.0353 & 59.4800 \\
\hline & 3 & 1.1000 & 100.0003 & 34.0500 & 1.0999 & 100.0024 & 28.5171 \\
\hline & 6 & 1.0271 & 52.1765 & 10.8259 & 1.0511 & 49.4929 & 19.2359 \\
\hline & 8 & 1.0862 & 0 & 24.9951 & 1.0924 & 0 & 24.9875 \\
\hline Total & & & 349.8529 & 169.5854 & & 344.4340 & 164.6120 \\
\hline \multirow[t]{8}{*}{ Discos } & 4 & 0.9983 & 110.1130 & 53.3301 & 1.0050 & 103.1062 & 49.9366 \\
\hline & 7 & 1.0456 & 0 & 0 & 1.0521 & 0 & 0 \\
\hline & 9 & 1.0343 & 5.0015 & 2.4223 & 1.0410 & 5.0037 & 2.4234 \\
\hline & 10 & 1.0151 & 13.8970 & 6.7306 & 1.0227 & 22.2554 & 10.7788 \\
\hline & 11 & 0.9939 & 28.4203 & 13.7646 & 1.0178 & 19.7659 & 9.5731 \\
\hline & 12 & 0.9700 & 31.4859 & 15.2493 & 0.9954 & 30.8817 & 14.9567 \\
\hline & 13 & 1.0027 & 5.0000 & 2.4216 & 1.0255 & 5.0174 & 2.4301 \\
\hline & 14 & 0.9905 & 20.1220 & 9.7455 & 1.0063 & 26.6867 & 12.9250 \\
\hline Total & & & 332.8384 & 161.2010 & & 328.0524 & 158.8830 \\
\hline
\end{tabular}

TABLE V. COMPARISON OF GA AND GWO SCHEDULING OF GENCOS AND DISCOS WITHOUT TCSC FOR THE IEEE 30 BUS SYSTEM

\begin{tabular}{|c|c|c|c|c|c|c|c|c|c|c|}
\hline & \multirow{2}{*}{$\begin{array}{c}\text { Bus } \\
\text { no }\end{array}$} & \multicolumn{3}{|c|}{ GA [22] } & \multicolumn{3}{|c|}{ GWO [22] } & \multicolumn{3}{|c|}{ COA } \\
\hline & & $\mathbf{V}(\mathbf{p u})$ & P (MW) & Q (Mvar) & $\mathrm{V}(\mathrm{pu})$ & P (MW) & Q (Mvar) & $\mathbf{V}(\mathbf{p u})$ & P (MW) & Q (Mvar) \\
\hline \multirow[t]{6}{*}{ Gen } & 1 & 1.0 & 180.0 & -40.2 & 1.0 & 178.0 & -39.78 & 1.0 & 192.5 & 8.253 \\
\hline & 2 & 1.0 & 53.19 & 54.31 & 1.0 & 60.29 & 52.42 & 0.977 & 79.67 & 37.74 \\
\hline & 5 & 1.0 & 35.32 & 89.76 & 1.0 & 33.61 & 91.46 & 0.947 & 32.56 & 70.01 \\
\hline & 8 & 1.0 & 35.0 & 53.43 & 1.0 & 34.99 & 53.21 & 0.953 & 19.66 & 54.63 \\
\hline & 11 & 1.0 & 30.0 & 12.69 & 1.0 & 30.0 & 12.09 & 0.997 & 27.48 & 15.84 \\
\hline & 13 & 1.0 & 40.0 & 28.27 & 1.0 & 40.0 & 32.06 & 0.999 & 39.35 & 20.74 \\
\hline Total & & & 373.5 & 198.05 & & 376.9 & 201.48 & & 391.3 & 207.2 \\
\hline \multirow[t]{21}{*}{ Dis } & 2 & 1.000 & 50.0 & 24.22 & 1.000 & 50.0 & 24.22 & 0.977 & 49.98 & 24.20 \\
\hline & 3 & 0.984 & 8.82 & 4.27 & 0.984 & 9.7 & 4.7 & 0.956 & 3.647 & 1.766 \\
\hline & 4 & 0.982 & 8.51 & 4.12 & 0.982 & 2.02 & 0.98 & 0.946 & 9.927 & 4.807 \\
\hline & 5 & 1.000 & 100.0 & 48.43 & 1.000 & 100.0 & 48.43 & 0.947 & 99.94 & 48.40 \\
\hline & 7 & 0.974 & 46.31 & 22.43 & 0.972 & 49.14 & 23.80 & 0.925 & 50.00 & 24.21 \\
\hline & 8 & 1.000 & 34.12 & 16.52 & 1.000 & 33.39 & 16.46 & 0.953 & 50.00 & 24.21 \\
\hline & 10 & 0.959 & 9.36 & 4.53 & 0.961 & 4.25 & 2.06 & 0.950 & 8.390 & 4.063 \\
\hline & 12 & 0.962 & 33.91 & 16.42 & 0.957 & 49.96 & 24.2 & 0.972 & 19.20 & 9.303 \\
\hline & 14 & 0.941 & 8.31 & 4.02 & 0.942 & 3.6 & 1.74 & 0.942 & 10.00 & 4.843 \\
\hline & 15 & 0.942 & 5.72 & 2.77 & 0.935 & 9.89 & 4.79 & 0.937 & 9.885 & 4.787 \\
\hline & 16 & 0.949 & 9.10 & 4.41 & 0.955 & 2.41 & 1.17 & 0.954 & 6.738 & 3.263 \\
\hline & 17 & 0.953 & 4.76 & 2.31 & 0.958 & 3.18 & 1.54 & 0.950 & 2.894 & 1.401 \\
\hline & 18 & 0.927 & 6.5 & 3.15 & 0.929 & 7.91 & 3.83 & 0.918 & 6.122 & 2.965 \\
\hline & 19 & 0.928 & 8.37 & 4.05 & 0.936 & 2.34 & 1.13 & 0.915 & 9.854 & 4.772 \\
\hline & 20 & 0.934 & 2.96 & 1.44 & 0.941 & 2.02 & 0.98 & 0.921 & 5.526 & 2.676 \\
\hline & 21 & 0.953 & 6.06 & 2.91 & 0.952 & 11.61 & 5.62 & 0.943 & 6.340 & 3.070 \\
\hline & 23 & 0.936 & 4.91 & 2.38 & 0.926 & 8.08 & 3.91 & 0.921 & 9.830 & 4.760 \\
\hline & 24 & 0.943 & 3.45 & 1.67 & 0.935 & 6.55 & 3.17 & 0.927 & 5.408 & 2.619 \\
\hline & 26 & 0.938 & 2.01 & 0.87 & 0.936 & 2.09 & 1.01 & 0.913 & 3.266 & 1.581 \\
\hline & 29 & 0.931 & 3.45 & 1.67 & 0.940 & 2.06 & 1.000 & 0.913 & 3.001 & 1.453 \\
\hline & 30 & 0.922 & 6.57 & 3.18 & 0.932 & 5.59 & 2.71 & 0.901 & 7.499 & 3.632 \\
\hline Total & & & 363.1 & 175.87 & & 366.38 & 177.45 & & 377.4 & 182.8 \\
\hline
\end{tabular}




\section{B. IEEE 30-Bus Test System}

The IEEE 30 bus data are considered from [31]. The system has 6 GENCOS and 21 DISCOS with 41 transmission lines, as shown in Figure 8. The marginal benefit function for GENCOS and DISCOS are given in [31]. The implementation of COA in scheduling GENCOS and DISCOS in the case without TCSC is presented in Table $\mathrm{V}$. The Table shows that the total real power of generator and total power consumption when using the COA algorithm are higher than when using GA and GWO algorithms [22].

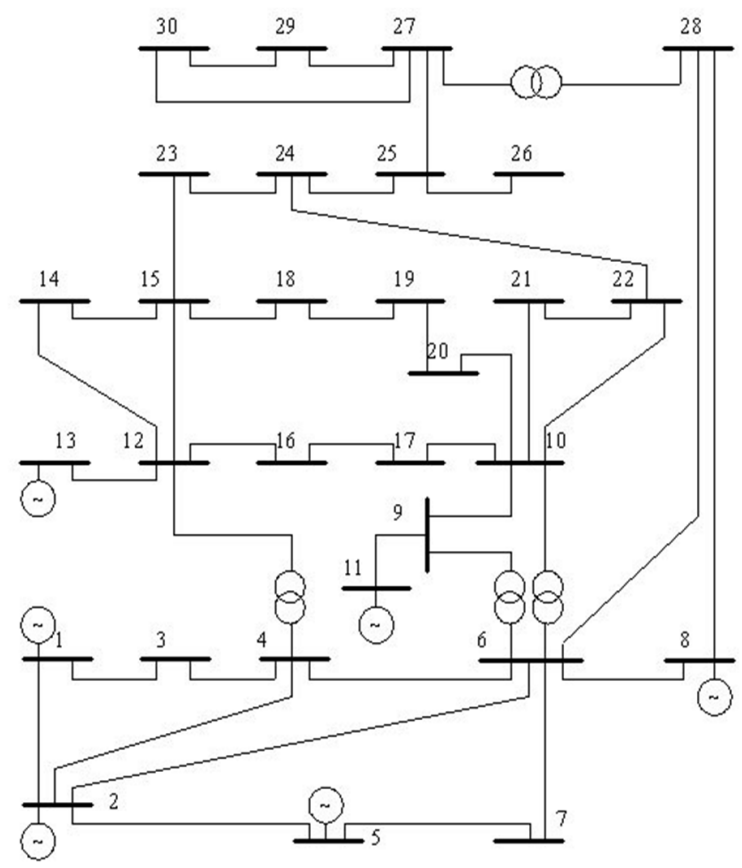

Fig. 8. The IEEE 30-bus system

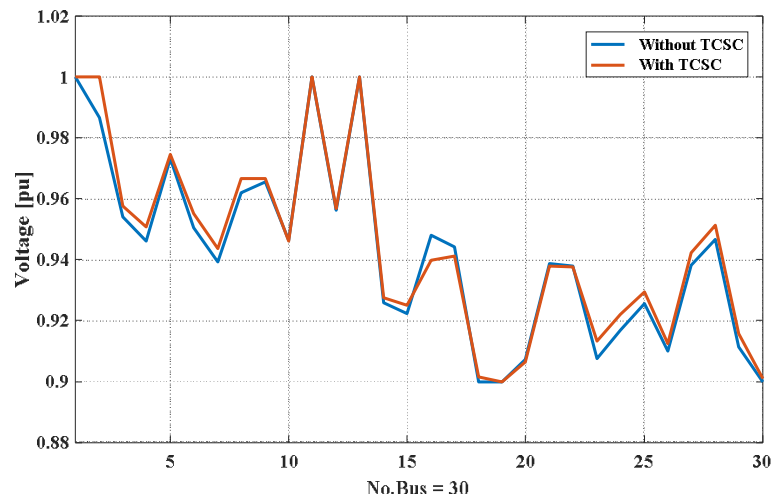

Fig. 9. The bus voltage profile of IEEE 30-bus system without and with TCSC

The scheduling is different reflecting in better social benefit, which is shown in Table VI. Using GA and GWO algorithm, the social benefit is $5098.72(\$ / \mathrm{h})$, and 5116.56 $(\$ / \mathrm{h})$, and that of COA is $5771.90(\$ / \mathrm{h})$, which is $11.66 \%$ and $11.35 \%$ higher respectively. Figures $9-10$ show the power on the branches and the voltage at the buses in the case without and with TCSC when using the COA algorithm, and they meet the allowable limits. It can be seen that, the social benefit value of using COA with TCSC is $5828.29(\$ / \mathrm{h})$ as shown in Table VI, which is $12.28 \%$ higher than the social benefit of using GA with TCSC, and is $6.66 \%$ higher than the social benefit of using GWO with TCSC. From Figures 11-12 it can be seen that in the case with TCSC, COA has the ability to converge quicker than PSO and BOA. This shows that the COA is one of the useful methods for TCSC installation to maximize social welfare.

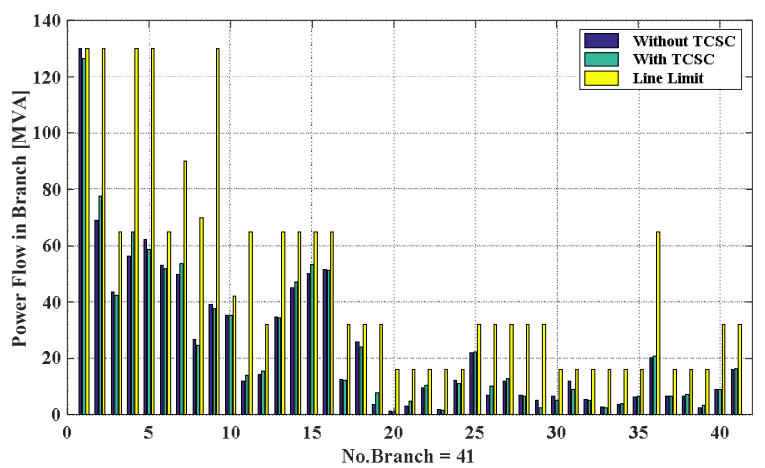

Fig. 10. Power flow branch of IEEE 30-bus system without and with TCSC

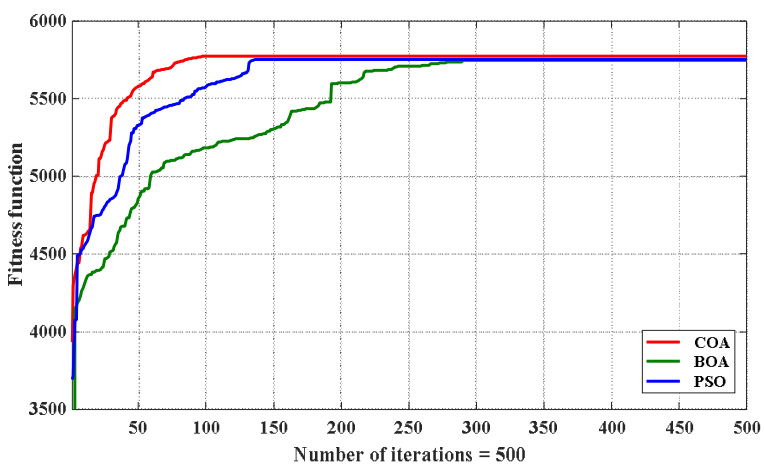

Fig. 11. Convergence characteristics of COA compared with PSO and BOA for the IEEE 30 bus system without TCSC

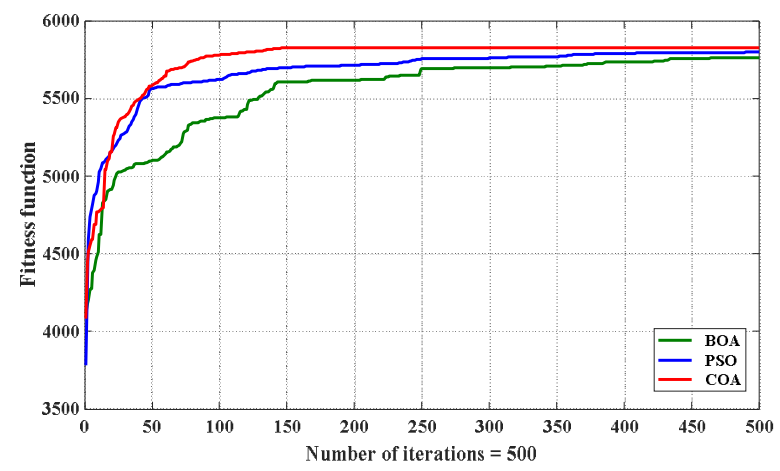

Convergence characteristics of COA compared with PSO and BOA for the IEEE 30 bus system with TCSC 
TABLE VI. COMPARISON OF GA, GWO AND COA FOR SOCIAL WELFARE MAXIMIZATION FOR THE IEEE 30 BUS SYSTEM

\begin{tabular}{|c|l|c|c|c|c|c|}
\hline \multirow{2}{*}{ COA } & \multicolumn{3}{|c|}{ Without TCSC } & \multicolumn{3}{c|}{ With TCSC } \\
\cline { 2 - 7 } & GA [22] & GWO [22] & COA & GA [22] & GWO [22] & COA \\
\hline Gencos & 1133.95 & 1144.84 & 1392.7028 & 1123.76 & 1250.19 & 1404.7130 \\
\hline Discos & 6232.68 & 6261.40 & 7164.8892 & 6235.99 & 6689.80 & 7233.0322 \\
\hline Social Welfare & 5098.72 & 5116.56 & 5771.9023 & 5112.22 & 5439.61 & 5828.2939 \\
\hline \multicolumn{3}{|l}{ Location of TCSC } & Line 6-28 & Line 6-8 & Line 1-2 \\
\hline
\end{tabular}

\section{CONCLUSIONS}

Maximizing social welfare is one of the most important issues in today's competitive electricity market and a difficult challenge for the operator system to implement. Maximizing social welfare depends on the optimal rescheduling of generation, demand levels, and optimal placement of TCSC. In order to solve this problem, an effective method is required. In this paper, the Cuckoo Optimization Algorithm was proposed to maximize social welfare via optimal placing and sizing of TCSC. The test results on the IEEE 14-bus and the IEEE 30bus systems when utilizing COA were compared with the results from GA and GWO. The obtained simulation results demonstrate that the COA is a useful method for TCSC installation to maximize social welfare.

\section{REFERENCESS}

[1] J. D. Weber, T. J. Overbye, "An individual welfare maximization algorithm for electricity markets", IEEE Transactions Power Systems, Vol. 17, No. 3, pp. 590-596, 2002

[2] H. Liu, Y. Shen, Z. B. Zabinsky, C. C. Liu, A. Courts, S. K. Joo, "Social welfare maximization in transmission enhancement considering network congestion", IEEE Transactions on Power Systems, Vol. 23, No. 3, pp. 1105-1114, 2008

[3] K. Singh, N. P. Padhy, J. D. Sharma, "Social welfare maximization considering reactive power and congestion management in the deregulated environment", Electric Power Components and System, Vol. 38, No. 1, pp. 50-71, 2010

[4] D. Gautam, N. Mithulananthan, "Locating distributed generator in the LMP-based electricity market for social welfare maximization", Electric Power Components and Systems, Vol. 35, No. 5, pp. 489-503, 2007

[5] P. S. Georgilakis, P. G. Vernados, "Flexible AC transmission system controllers: An evaluation", Materials Science Forum, Vol. 670, pp. 399406, 2011

[6] M. Y. A. Khan, U. Khalil, H. Khan, A. Uddin, S. Ahmed, "Power flow control by unified power flow controller", Engineering, Technology \& Applied Science Research, Vol. 9, No. 2, pp. 3900-3904, 2019

[7] Z. M. Zohrabad, "Application of hybrid HS and tabu search algorithm for optimal location of facts devices to reduce power losses in power systems", Engineering, Technology \& Applied Science Research, Vol. 6, No. 6, pp. 1217-1220, 2016

[8] S. K. Verma, S. N. Singh, H. O. Gupta, "Location of unified power flow controller for congestion management", Electric Power Systems Research, Vol. 58, No. 2, pp. 89-96, 2001

[9] S. N. Singh, A. K. David, "Optimal location of facts devices for congestion management”, Electric Power Systems Research, Vol. 58, No. 2, pp. 71-79, 2001

[10] N. Acharya, N. Mithulananthan, "Locating series facts devices for congestion management in deregulated electricity markets", Electric Power Systems Research, Vol. 77, No. 3-4, pp. 352-360, 2007

[11] K. Soleimani, J. Mazloum, "Considering facts in optimal transmission expansion planning", Engineering, Technology \& Applied Science Research, Vol. 7, No. 5, pp. 1987-1995, 2017
[12] G. B. Shrestha, W. Feng, "Effects of series compensation on spot price power markets", International Journal of Electric Power \& Energy Systems, Vol. 27, No. 5, pp. 428-436, 2005

[13] T. L. Duong, Y. J. Gang, L. A. T. Nguyen, G. Z. Wei, "Enhancing total transfer capability via optimal location of TCSC in deregulated electricity market", Recent Advances in Electrical Engineering and Related Sciences, Vol. 282, pp. 47-56, 2013

[14] T. L. Duong, Y. J. Gang, K. Tong, "Optimal location of thyristorcontrolled-series-capacitor using min cut algorithm", TELKOMNIKA Indonesian Journal of Electrical Engineering, Vol. 12, No. 5, pp. 36493661,2014

[15] T. L. Duong, Y. J. Gang, T. V. Anh, “Application of min cut algorithm for optimal location of facts devices considering system loadability and cost of installation", International Journal of Electrical Power \& Energy Systems, Vol. 63, pp. 979-987, 2014

[16] T. L. Duong, Y. J. Gang, V. A. Truong, "Improving the transient stability-constrained optimal power flow with thyristor controlled series capacitor", Russian Electrical Engineering, Vol. 85, No. 12, pp. 777784,2014

[17] T. L. Duong, Y. J. Gang, V. A. Truong, “A new method for secured optimal power flow under normal and network contingencies via optimal location of TCSC", International Journal of Electrical Power \& Energy Systems, Vol. 52, pp. 68-80, 2013

[18] T. Kang, J. G. Yao, T. L. Duong, S. J. Yang, X. Q. Zhu, "A hybrid approach for power system security enhancement via optimal installation of flexible AC transmission system (facts) devices", Energies, Vol. 10, No. 9, pp. 1305-1337, 2017

[19] D. T. Long, N. H. Q. Viet, V. A. Truong, V. T. Kien, “Optimal location of facts devices for congestion management and loadability enhancement", Journal Electrical Systems, Vol. 13. No. 3, pp. 579-594, 2017

[20] W. M. Lin, S. J. Che, Y. S. Su, "An application of interior-point based OPF for system expansion with facts devices in a deregulated environment", IEEE International Conference on Power System Technology, Perth, Australia, December 4-7, 2000

[21] Z. Yu, D. Lusan, "Optimal placement of facts devices in deregulated systems considering line losses", International Journal of Electrical Power \& Energy Systems, Vol. 26, No 10, pp. 813-819, 2004

[22] S. K. Behera, N. K. Mohanty, "Social welfare maximization with thyristor-controlled series compensator using grey wolf optimization algorithm", The International Journal of Electrical Engineering \& Education, Vol. 56, No. 1, pp. 1-14, 2019

[23] S. H. Song, J. U. Lim, S. Moon, "Installation and operation of facts devices for enhancing steady-state security", Electric Power Systems Research, Vol. 70, No. 1, pp. 7-15, 2004

[24] S. M. H. Nabavi, A. Kazemi, M. A. S. Masoum, "Social welfare maximization with fuzzy based genetic algorithm by TCSC and SSSC in double-sided auction market", Scientia Iranica, Vol. 19, No. 3, pp. 745758, 2012

[25] S. M. H. Nabavi, N. A. Hosseinipoor, "Social welfare maximization by optimal locating and sizing of TCSC for congestion management in deregulated power markets", International Conference on Power System Technology, Hangzhou, China, October 24-28, 2010

[26] K. Balamurugan, R. Muralisachithanandam, V. Dharmalingam, "Performance comparison of evolutionary programming and differential evolution approaches for social welfare maximization by placement of multi type facts devices in pool electricity market", International Journal of Electrical Power \& Energy Systems, Vol. 67, pp. 517-528, 2015 
[27] R. Rajabioun, "Cuckoo optimization algorithm", Applied Soft Computing, Vol. 11, No. 8, pp. 5508-5518, 2011

[28] T. N. L. Anh, D. N. Vo, W. Ongsakul, P. Vasant, T. Ganesan, "Cuckoo optimization algorithm for optimal power flow", 18th Asia Pacific Symposium on Intelligent and Evolutionary Systems, Singapore, November 10-12, 2015

[29] L. L. Lai, Power system restructuring and deregulation, John Wiley and Sons, 2001

[30] R. Zimmerman, D. Gan, MATPOWER: A matlab power system simulation package, Cornell University, 2016

[31] T. Pend, K. Tomsovic, "Congestion influence on bidding strategies in an electricity market”, IEEE Transactions on Power Systems, Vol. 18, No. 3, pp. 1054-1061, 2003 